\title{
CONTINUOUS DEPENDENCE OF SOLUTIONS OF STOCHASTIC DIFFERENTIAL EQUATIONS DRIVEN BY STANDARD AND FRACTIONAL BROWNIAN MOTION ON A PARAMETER
}

UDC 519.21

\author{
YU. S. MISHURA, S. V. POSASHKOVA, AND S. V. POSASHKOV
}

\begin{abstract}
We consider a stochastic differential equation driven by both a Wiener process and a fractional Brownian motion. The coefficients of the equation are nonhomogeneous, and the initial condition is random. It is assumed that both the coefficients and the initial condition depend on a parameter. We establish conditions on the coefficients and the initial condition for the continuous dependence of a solution on the parameter.
\end{abstract}

\section{INTRODUCTION}

In this paper, we study a stochastic differential equation defined on a certain stochastic basis $\left(\Omega, \mathcal{F},\left(\mathcal{F}_{t}, t \in[0, T]\right), \mathrm{P}\right)$. The coefficients of the equation depend on a parameter $u$, namely:

$$
\begin{gathered}
X_{t}^{u}=X_{0}^{u}+\int_{0}^{t} a^{u}\left(s, X_{s}^{u}\right) d s+\int_{0}^{t} b^{u}\left(s, X_{s}^{u}\right) d W_{s}+\int_{0}^{t} c^{u}\left(s, X_{s}^{u}\right) d B_{s}^{H}, \\
t \in[0, T]
\end{gathered}
$$

where $X_{0}^{u}$ is an $\mathcal{F}_{0}$-measurable random variable such that $\mathrm{E}\left(X_{0}^{u}\right)^{2}<\infty$ for all $u \in\left[0, u_{0}\right]$, $W=\left(W_{t}, \mathcal{F}_{t}, t \in[0, T]\right)$ is a standard Brownian motion, and $B^{H}=\left(B_{t}^{H}, \mathcal{F}_{t}, t \in[0, T]\right)$ is a fractional Brownian motion with the Hurst parameter $H \in(1 / 2,1)$. The coefficients $a^{u}, b^{u}, c^{u}:[0, T] \times \mathbb{R} \rightarrow \mathbb{R}$ are nonrandom measurable functions.

For the case of $H \in(3 / 4,1)$, the conditions on the coefficients are found in [2] under which the stochastic differential equation

$$
X_{t}=X_{0}+\int_{0}^{t} a\left(s, X_{s}\right) d s+\int_{0}^{t} b\left(s, X_{s} d W_{s}+\int_{0}^{t} c\left(s, X_{s}\right) d B_{s}^{H}, \quad t \in[0, T],\right.
$$

has a unique solution whose trajectories belong to a Besov space. It is also proved in [2] that the solution possesses the Hölder property of an order that is less than $1 / 2$. In what follows we a priori assume that a solution of (2) exists for the case of $H \in(1 / 2,3 / 4$ ] and moreover we assume that the solution has the same properties as in the case of $H \in(3 / 4,1)$.

Turning to the equation depending on a parameter, we assume that the stochastic differential equation has a unique solution whose trajectories belong to a Besov space for

2010 Mathematics Subject Classification. Primary 60G22; Secondary 60H10.

Key words and phrases. Fractional Brownian motion, standard Brownian motion, stochastic differential equation, continuity in a parameter. 
every value of the parameter and that this solution possesses the Hölder property of an order that is less than $1 / 2$.

For this equation, we find conditions imposed on the coefficients and on the initial condition as functions of the parameter under which the solution, as a function of the same parameter, converges in probability to a solution of the stochastic differential equation whose coefficients and the initial value are the limits of the coefficients and initial value, respectively, of the prelimit equation.

Some results concerning the existence and uniqueness of a solution of a stochastic differential equation driven by both a standard Brownian motion and a fractional Brownian motion are given in Section 1 .

Conditions are found in Section 2 such that a solution of a stochastic differential equation driven by both a standard Brownian motion and a fractional Brownian motion continuously depends on a parameter for the case where the coefficients are nonhomogeneous and the initial condition is random.

\section{EXISTENCE AND UNIQUENESS OF A SOLUtion}

We consider the following stochastic differential equation defined on a certain complete stochastic basis $\left(\Omega, \mathcal{F},\left(\mathcal{F}_{t}, t \in[0, T]\right), \mathrm{P}\right)$ :

$$
X_{t}=X_{0}+\int_{0}^{t} a\left(s, X_{s}\right) d s+\int_{0}^{t} b\left(s, X_{s}\right) d W_{s}+\int_{0}^{t} c\left(s, X_{s}\right) d B_{s}^{H}, \quad t \in[0, T],
$$

where $X_{0}$ is an $\mathcal{F}_{0}$-measurable random variable such that $\mathrm{E} X_{0}^{2}<\infty, W=\left(W_{t}, \mathcal{F}_{t}, t \in\right.$ $[0, T])$ is a standard Brownian motion, and $B^{H}=\left(B_{t}^{H}, \mathcal{F}_{t}, t \in[0, T]\right)$ is a fractional Brownian motion (fBm) with the Hurst parameter $H \in(1 / 2,1)$. The coefficients $a, b, c$ : $[0, T] \times \mathbb{R} \rightarrow \mathbb{R}$ are nonrandom measurable nonhomogeneous functions.

Assume that the following conditions hold.

(A) There exists a constant $A>0$ such that

$$
|a(t, x)| \leq A, \quad|b(t, x)| \leq A, \quad|c(t, x)| \leq A
$$

for all $t \in[0, T]$ and $x \in \mathbb{R}$.

(B) There exists a constant $L>0$ such that

$$
(a(t, x)-a(t, y))^{2}+(b(t, x)-b(t, y))^{2}+(c(t, x)-c(t, y))^{2} \leq L^{2}(x-y)^{2}
$$

for all $t \in[0, T]$ and $x, y \in \mathbb{R}$.

(C) The function $c(t, x)$ is differentiable with respect to $x$; there are constants $B>0$ and $\beta \in(1-H, 1)$ such that

$$
|c(s, x)-c(t, x)|+\left|\partial_{x} c(s, x)-\partial_{x} c(t, x)\right| \leq B|s-t|^{\beta}
$$

for all $s, t \in[0, T]$ and $x \in \mathbb{R}$.

(D) The derivative $\partial_{x} c(t, x)$ with respect to $x$ is such that

$$
\left|\partial_{x} c(t, x)-\partial_{x} c(t, y)\right| \leq D|x-y|^{\rho}
$$

for all $t \in[0, T]$ and $x, y \in \mathbb{R}$, where the $\rho \in(3 / 2-H, 1)$ is fixed.

For some $1-H<\alpha<\min (\beta, \rho-1 / 2)$, consider a Besov type space

$$
W_{\alpha}([0, T]):=\left\{Y=Y_{t}(\omega):(t, \omega) \in[0, T] \times \Omega,\|Y\|_{\alpha}<\infty\right\}
$$

equipped with the norm

$$
\|Y\|_{\alpha}^{2}:=\sup _{t \in[0, T]}\left(\mathrm{E}\left(Y_{t}\right)^{2}+\mathrm{E}\left(\int_{0}^{t} \frac{\left|Y_{t}-Y_{s}\right|}{(t-s)^{1+\alpha}} d s\right)^{2}\right) .
$$


The following result is proved in [2] for $H \in(3 / 4,1)$; the proof presented in [2] is correct for $H \in(1 / 2,3 / 4]$, too.

Theorem 1 ([2]). The stochastic differential equation (3) has a unique solution in the interval $[0, T]$, and its trajectories belong to the space $W_{\alpha}([0, T])$. Moreover, the solution possesses the Hölder property with an order that is less than $1 / 2$.

\section{Convergence of solutions in probability}

Now we consider a stochastic differential equation of the form (3) with a parameter $u \in\left[0, u_{0}\right]:$

(7) $X_{t}^{u}=X_{0}^{u}+\int_{0}^{t} a^{u}\left(s, X_{s}^{u}\right) d s+\int_{0}^{t} b^{u}\left(s, X_{s}^{u}\right) d W_{s}+\int_{0}^{t} c^{u}\left(s, X_{s}^{u}\right) d B_{s}^{H}, \quad t \in[0, T]$,

where $X_{0}^{u}$ is an $\mathcal{F}_{0}$-measurable random variable such that $\mathrm{E}\left(X_{0}^{u}\right)^{2}<\infty$ for all $u \in$ $\left[0, u_{0}\right], W=\left(W_{t}, \mathcal{F}_{t}, t \in[0, T]\right)$ is a standard Brownian motion, and $B^{H}=\left(B_{t}^{H}, \mathcal{F}_{t}, t \in\right.$ $[0, T])$ is a fractional Brownian motion with the Hurst parameter $H \in(1 / 2,1)$. The coefficients $a^{u}, b^{u}, c^{u}:[0, T] \times \mathbb{R} \rightarrow \mathbb{R}$ are nonrandom measurable functions. Assume that the coefficients of this equation satisfy conditions similar to (A)-(D).

(A1) There exists a constant $A>0$ such that

$$
\left|a^{u}(t, x)\right| \leq A, \quad\left|b^{u}(t, x)\right| \leq A, \quad\left|c^{u}(t, x)\right| \leq A
$$

for all $t \in[0, T]$ and $x \in \mathbb{R}$.

(B1) There exists a constant $L>0$ such that

$$
\left(a^{u}(t, x)-a^{u}(t, y)\right)^{2}+\left(b^{u}(t, x)-b^{u}(t, y)\right)^{2}+\left(c^{u}(t, x)-c^{u}(t, y)\right)^{2} \leq L^{2}(x-y)^{2}
$$

for all $t \in[0, T]$ and $x, y \in \mathbb{R}$.

(C1) The function $c^{u}(t, x)$ is differentiable with respect to $x$, and there are constants $B>0$ and $\beta \in(1-H, 1)$ such that

$$
\left|c^{u}(s, x)-c^{u}(t, x)\right|+\left|\partial_{x} c^{u}(s, x)-\partial_{x} c^{u}(t, x)\right| \leq B|s-t|^{\beta}
$$

for all $s, t \in[0, T]$ and $x \in \mathbb{R}$.

(D1) The derivative $\partial_{x} c^{u}(t, x)$ with respect to $x$ is such that

$$
\left|\partial_{x} c^{u}(t, x)-\partial_{x} c^{u}(t, y)\right| \leq D|x-y|^{\rho}
$$

for all $t \in[0, T]$ and $x, y \in \mathbb{R}$, where $\rho \in(3 / 2-H, 1)$ is fixed.

We apply Theorem 1 to equation (7) if $H \in(3 / 4,1)$. Otherwise, if $H \in(1 / 2,3 / 4]$, we show that the equation has a Hölderian solution of an order that is less than $1 / 2$.

Let $\alpha \in(0,1)$. Consider the space $W_{0}^{\alpha, 1}(0, T)$ of measurable functions $f:[0, T] \rightarrow \mathbb{R}$ such that

$$
\int_{0}^{T} \frac{|f(t)|}{t^{\alpha}} d t+\int_{0}^{T} \int_{0}^{t} \frac{|f(t)-f(s)|}{(t-s)^{1+\alpha}} d s d t<+\infty .
$$

The left and right Riemann-Liouville fractional integrals of order $\alpha$ are defined for functions $f \in L^{1}(0, T)$ and for almost all $t \in(0, T)$ as follows:

$$
\begin{gathered}
I_{0+}^{\alpha} f(t):=\frac{1}{\Gamma(\alpha)} \int_{0}^{t} f(s)(t-s)^{\alpha-1} d s \\
I_{T-}^{\alpha} f(t):=\frac{(-1)^{-\alpha}}{\Gamma(1-\alpha)} \int_{t}^{T} f(s)(s-t)^{\alpha-1} d s
\end{gathered}
$$

(see [4]). Let $I_{0+}^{\alpha}\left(L^{1}(0, T)\right)\left(I_{T-}^{\alpha}\left(L^{1}(0, T)\right)\right)$ be the image of $L^{1}(0, T)$ under the action of the operator $I_{0+}^{\alpha}\left(I_{T-}^{\alpha}\right)$. The restriction of the function $f \in W_{0}^{\alpha, 1}(0, T)$ to $(0, t)$ belongs 
to the space $I_{0+}^{\alpha}\left(L^{1}(0, t)\right)$ for almost all $t$. If $f \in I_{0+}^{\alpha}\left(L^{1}\right)\left(f \in I_{T-}^{\alpha}\left(L^{1}\right)\right)$ and $0<\alpha<1$, then the Weil derivatives,

$$
D_{0+}^{\alpha} f(t):=\frac{1}{\Gamma(1-\alpha)}\left(\frac{f(t)}{t^{\alpha}}+\alpha \int_{0}^{t} \frac{f(t)-f(s)}{(t-s)^{\alpha+1}} d s\right) \mathbb{I}_{(0, T)}(t)
$$

(right Weil derivative) and

$$
D_{T-}^{\alpha} f(t):=\frac{(-1)^{\alpha}}{\Gamma(1-\alpha)}\left(\frac{f(t)}{(T-t)^{\alpha}}+\alpha \int_{t}^{T} \frac{f(t)-f(s)}{(s-t)^{\alpha+1}} d s\right) \mathbb{I}_{(0, T)}(t)
$$

(left Weil derivative), are defined for almost all $t \in(0, T)$ (see [4]). The convergence of the integrals at the point of singularity $t=s$ is understood pointwise and holds for almost all $t \in(0, T)$. In what follows we put $f_{a+}(x)=(f(x)-f(a+)) I_{(a, b)}(x)$ and $f_{b-}(x)=(f(b-)-f(x)) I_{(a, b)}(x)$.

The following result is obtained in [1].

Lemma 1. Let $B^{H}=\left(B_{t}^{H}, \mathcal{F}_{t}, t \in[0, T]\right)$ be a fractional Brownian motion with the Hurst parameter $H \in(1 / 2,1)$. If $1-H<\alpha<1 / 2$, then

$$
\mathrm{E} \sup _{0 \leq s \leq t \leq T}\left|D_{t-}^{1-\alpha} B_{t-}^{H}(s)\right|^{p}<\infty
$$

for all $T>0$ and $p \in[1, \infty)$.

Lemma 1 implies that if a function $f$ belongs to the class $W_{0}^{\alpha, 1}(0, T)$ with $1-H<$ $\alpha<1 / 2$ and satisfies inequality (9), then the integral with respect to the fractional Brownian motion is defined by

$$
\int_{0}^{t} f(s) d B_{s}^{H}=\int_{0}^{t} D_{0+}^{\alpha} f(s) D_{t-}^{1-\alpha} B_{t-}^{H}(s) d s .
$$

The integral is estimated as follows:

$$
\left|\int_{0}^{t} f(s) d B_{s}^{H}\right| \leq C_{t}(\omega)\left(\int_{0}^{t} \frac{|f(s)|}{s^{\alpha}} d s+\int_{0}^{t} \int_{0}^{r} \frac{|f(r)-f(u)|}{(r-u)^{1+\alpha}} d u d r\right)
$$

(see [1]), where

$$
C_{t}(\omega)=\sup _{0 \leq u \leq s \leq t}\left|D_{s-}^{1-\alpha} B_{s-}^{H}(u)\right|<\infty .
$$

It is proved in [2], Section 3.2, that the process $C_{t}(\omega)$ is bounded from above, namely

$$
C_{t}(\omega) \leq C_{\varepsilon} \psi_{t}
$$

where the stochastic process $\psi_{t}$ is of the form

$$
\psi_{t}:=\left(\int_{0}^{t} \int_{0}^{t} \frac{\left|B_{w}^{H}-B_{v}^{H}\right|^{2 / \varepsilon}}{|w-v|^{2 H / \varepsilon}} d w d v\right)^{\varepsilon / 2}
$$

for $0<\varepsilon<H+\alpha-1$ and where $C_{\varepsilon}$ is a constant depending on $\varepsilon$. It is clear that the process $\left(\psi_{t}, t \in[0, T]\right)$ is continuous and increasing with probability one.

Let $b_{v}^{u}=b^{u}\left(v, X_{v}^{u}\right)$. Given $0<\delta<1 / 2$ we choose $p>1 / \delta$ and

$$
\varrho=\frac{1}{p}\left(1+\frac{p}{2}-p \delta\right) .
$$

Let $0 \leq s \leq t \leq T$. We apply the Garsia-Rademich-Rumsey inequality to the function $f(t)=\int_{0}^{t} b_{v}^{u} d W_{v}$ (see, for example, [1]). This inequality for a continuous function $g$ reads as follows:

$$
|g(t)-g(s)|^{p} \leq C_{\varrho, p}|t-s|^{\varrho p-1} \int_{0}^{t} \int_{0}^{t} \frac{|g(x)-g(y)|^{p}}{|x-y|^{\varrho p+1}} d x d y .
$$


Taking into account (12) we see that

$$
\left|\int_{s}^{t} b_{v}^{u} d W_{v}\right| \leq C_{\varrho, p}|t-s|^{\varrho-1 / p} \xi_{t}\left(b^{u}\right) \leq C_{\varrho, p}|t-s|^{1 / 2-\delta} \xi_{t}\left(b^{u}\right)
$$

where

$$
\xi_{t}\left(b^{u}\right):=\left(\int_{0}^{t} \int_{0}^{t} \frac{\left|\int_{x}^{y} b_{v}^{u} d W_{v}\right|^{p}}{|x-y|^{\varrho p+1}} d x d y\right)^{1 / p} .
$$

Note that the process $\xi_{t}\left(b^{u}\right)$ is continuous and increasing.

For all $R>1$, we define a stopping time $\tau_{R, \delta}^{u, 0}$ by

$$
\tau_{R, \delta}^{u, 0}:=\inf \left\{t: C_{t}^{u}(\omega, \delta) \geq R\right\} \wedge T,
$$

where

$$
C_{t}^{u}(\omega, \delta):=\psi_{t} \vee \xi_{t}\left(b^{u}\right) \vee \xi_{t}\left(b^{0}\right) \vee 1
$$

In what follows the symbol $C$ stands for any constant that may depend on $T$ and on constants involved in conditions (A1)-(D1) but does not depend on $u$ and $R$. Let $\tau^{u}:=\tau_{R, \delta}^{u, 0}$.

Lemma 2. Assume that conditions (A1)-(D1) hold. Then $\mathrm{P}\left(\tau^{u}<T\right)$ is bounded from above and the bound does not depend on $u$ and tends to 0 as $R \rightarrow+\infty$.

Proof. For $R>1$, consider

$$
\begin{aligned}
\mathrm{P}\left(\tau^{u}<T\right) & \leq \mathrm{P}\left(C_{T}^{u}(\omega, \delta) \geq R\right) \\
& \leq \mathrm{P}\left(\xi_{T}\left(b^{u}\right) \geq R\right)+\mathrm{P}\left(\xi_{T}\left(b^{0}\right) \geq R\right)+\mathrm{P}\left(\psi_{T} \geq R\right) .
\end{aligned}
$$

Note that $\mathrm{P}\left(\psi_{T} \geq R\right)$ does not depend on $u$ and tends to 0 as $\mathbb{R} \rightarrow+\infty$, since all the moments exist for the process $\left(\psi_{t}, t \in[0, T]\right)$.

It is clear that $\mathrm{P}\left(\xi_{T}\left(b^{u}\right) \geq R\right) \leq R^{-1} \mathrm{E} \xi_{T}\left(b^{u}\right)$. Choose some constant $q>p>1$. Then

$$
\begin{aligned}
\mathrm{E} \xi_{T}\left(b^{u}\right) & \leq\left(\mathrm{E}\left(\xi_{T}\left(b^{u}\right)\right)^{q}\right)^{1 / q} \\
& =\left(\mathrm{E}\left(\int_{0}^{T} \int_{0}^{T} \frac{\left|\int_{x}^{y} b_{v}^{u} d W_{v}\right|^{p}}{|x-y|^{\varrho p+1}} d x d y\right)^{q / p}\right)^{1 / q} \\
& \leq\left(\int_{0}^{T} \int_{0}^{T} \frac{\mathrm{E}\left|\int_{x}^{y} b_{v}^{u} d W_{v}\right|^{q} d x d y}{|x-y|^{(\varrho p+1-\theta) q / p}}\right)^{1 / q}\left(\int_{0}^{T} \int_{0}^{T} \frac{d x d y}{|x-y|^{\theta q /(q-p)}}\right)^{(q-p) /(q p)},
\end{aligned}
$$

where we take $\theta \in(1-p / q+p(\varrho-1 / 2), 1-p / q)$ (note that $\varrho \leq 1 / 2)$. Thus

$$
\theta \frac{q}{q-p}<1
$$

and the second integral converges and is bounded by some constant in $[0, T]$.

Further, the first integral is estimated with the help of the Burkholder inequality as follows:

$$
\begin{aligned}
C\left(\int_{0}^{T} \int_{0}^{T} \frac{\mathrm{E}\left|\int_{x}^{y}\left(b_{v}^{u}\right)^{2} d v\right|^{q / 2} d x d y}{|x-y|^{(\varrho p+1-\theta) q / p}}\right)^{1 / q} & \leq C A\left(\int_{0}^{T} \int_{0}^{T}|x-y|^{q / 2-(\varrho p+1-\theta) q / p} d x d y\right)^{1 / q} \\
& \leq C A,
\end{aligned}
$$

since $q / 2-(\varrho p+1-\theta) q / p>q / 2-(\varrho p+1-1+p / q-p(\varrho-1 / 2)) q / p=-1$. Thus $\mathrm{P}\left(\xi_{T}\left(b^{u}\right) \geq R\right) \leq R^{-1} C A$ and the bound does not depend on the parameter $u$.

The proof of $\mathrm{P}\left(\xi_{T}\left(b^{0}\right) \geq R\right) \leq R^{-1} C A$ is similar. 
In what follows we need the following modification of Lemma 7.1 of [1]. The difference between Lemma 7.1 in 1 and Lemma 3 below is that the spatial variable belongs to $\mathbb{R}$ in Lemma 3. while it belongs to a bounded ball in Lemma 7.1 of [1]. However this difference does not influence the proof.

Lemma 3. Let $\sigma:[0, T] \times \mathbb{R} \longrightarrow \mathbb{R}$ be a function such that $\sigma(t, x)$ is differentiable with respect to $x$ and there are some constants $0<\beta, \delta \leq 1$ and $M_{0}, M>0$ for which the following conditions hold:

1) Lipschitz property with respect to $x$ :

$$
|\sigma(t, x)-\sigma(t, y)| \leq M_{0}|x-y|
$$

for all $t \in[0, T]$ and $x, y \in \mathbb{R}$.

2) Hölder property with respect to $x$ :

$$
\left|\partial_{x} \sigma(t, x)-\partial_{x} \sigma(t, y)\right| \leq M|x-y|^{\delta}
$$

for all $t \in[0, T]$ and $x, y \in \mathbb{R}$.

3) Hölder property with respect to $t$ :

$$
|\sigma(t, x)-\sigma(s, x)|+\left|\partial_{x} \sigma(t, x)-\partial_{x} \sigma(s, x)\right| \leq M_{0}|t-s|^{\beta}
$$

for all $t, s \in[0, T]$ and $x \in \mathbb{R}$.

Then

$$
\begin{aligned}
& \left|\sigma\left(t_{1}, x_{1}\right)-\sigma\left(t_{2}, x_{2}\right)-\sigma\left(t_{1}, x_{3}\right)+\sigma\left(t_{2}, x_{4}\right)\right| \\
& \leq M_{0}\left|x_{1}-x_{2}-x_{3}+x_{4}\right|+M_{0}\left|x_{1}-x_{3}\right| \cdot\left|t_{2}-t_{1}\right|^{\beta} \\
& \quad+M\left|x_{1}-x_{3}\right|\left(\left|x_{1}-x_{2}\right|^{\delta}+\left|x_{3}-x_{4}\right|^{\delta}\right)
\end{aligned}
$$

for all $x_{1}, x_{2}, x_{3}, x_{4} \in \mathbb{R}$.

Theorem 2. Assume that conditions (A1)-(D1) hold. Let

$$
d^{u}(t, x):=c^{u}(t, x)-c^{0}(t, x), \quad t \in[0, T], x \in \mathbb{R}, u \in\left[0, u_{0}\right] .
$$

Assume that the coefficients of the stochastic differential equation (7) satisfy the following conditions.

(G0) $X_{0}^{u} \rightarrow X_{0}^{0}$ in probability as $u \rightarrow 0$.

(G1) There are $\alpha^{u}>0, \beta^{u}>0, \gamma^{u}>0$, and $\alpha<\nu_{1} \leq 1$ such that

$$
\begin{aligned}
& \left|a^{u}(t, x)-a^{0}(t, x)\right| \leq \alpha^{u}\left(1+|x|^{\nu_{1}}\right), \\
& \left|b^{u}(t, x)-b^{0}(t, x)\right| \leq \beta^{u}\left(1+|x|^{\nu_{1}}\right), \\
& \left|c^{u}(t, x)-c^{0}(t, x)\right| \leq \gamma^{u}\left(1+|x|^{\nu_{1}}\right)
\end{aligned}
$$

for all $t \in[0, T]$ and $x \in \mathbb{R} ;$ moreover $\alpha^{u} \rightarrow 0, \beta^{u} \rightarrow 0$, and $\gamma^{u} \rightarrow 0$ as $u \rightarrow 0$.

(G2) There are constants $\varphi^{u}>0, \varkappa^{u}>0, \alpha<\nu_{2} \leq 1, \theta_{1}>\alpha$, and $\theta_{2}>2 \alpha$ such that

$$
\begin{gathered}
\left|d^{u}(t, x)-d^{u}(s, x)\right| \leq \varphi^{u}|t-s|^{\theta_{1}}\left(1+|x|^{\nu_{2}}\right), \\
\left|d^{u}(t, x)-d^{u}(t, y)\right| \leq \varkappa^{u}|x-y|^{\theta_{2}},
\end{gathered}
$$

for all $t, s \in[0, T]$ and $x, y \in \mathbb{R} ;$ moreover $\varphi^{u} \rightarrow 0$ and $\varkappa^{u} \rightarrow 0$ as $u \rightarrow 0$.

Then the sequence of solutions $\left\{X_{t}^{u}, t \in[0, T], u \in\left[0, u_{0}\right]\right\}$ converges to $\left\{X_{t}^{0}, t \in[0, T]\right\}$ uniformly in probability as $u \rightarrow 0$.

Proof. Fix an arbitrary $\varepsilon>0$. As above, $\tau^{u}:=\tau_{R, \delta}^{u, 0}$. Then

$$
\mathrm{P}\left(\sup _{t \in[0, T]}\left|X_{t}^{u}-X_{t}^{0}\right|>\varepsilon\right) \leq \mathrm{P}\left(\tau^{u}<T\right)+\mathrm{P}\left(\sup _{t \in[0, T]}\left|X_{t}^{u}-X_{t}^{0}\right|>\varepsilon, \tau^{u}=T\right) .
$$


Lemma 2 implies that the first probability on the right hand side of (16) is bounded and the bound does not depend on $u$ and tends to 0 as $R \rightarrow+\infty$.

Consider the second probability on the right hand side of (16). In view of (G0), we may assume that $X_{0}^{u}=X_{0}^{0}=0$. Further,

$$
\mathrm{P}\left(\sup _{t \in[0, T]}\left|X_{t}^{u}-X_{t}^{0}\right|>\varepsilon, \tau^{u}=T\right) \leq \mathrm{P}\left(\sup _{t \in[0, T]}\left|X_{t \wedge \tau^{u}}^{u}-X_{t \wedge \tau^{u}}^{0}\right|>\varepsilon\right) .
$$

Applying Chebyshev's inequality we get

$$
\mathrm{P}\left(\sup _{t \in[0, T]}\left|X_{t \wedge \tau^{u}}^{u}-X_{t \wedge \tau^{u}}^{0}\right|>\varepsilon\right) \leq \frac{1}{\varepsilon^{2}} \mathrm{E}\left(\sup _{t \in[0, T]}\left|X_{t \wedge \tau^{u}}^{u}-X_{t \wedge \tau^{u}}^{0}\right|\right)^{2}
$$

Given an arbitrary $z \in[0, T]$, consider the following sum:

$$
\begin{aligned}
& \mathrm{E}\left(\sup _{t \in[0, z]}\left|X_{t \wedge \tau^{u}}^{u}-X_{t \wedge \tau^{u}}^{0}\right|\right)^{2}+\mathrm{E}\left(\int_{0}^{z \wedge \tau^{u}} \frac{\left|X_{z \wedge \tau^{u}}^{u}-X_{z \wedge \tau^{u}}^{0}-X_{s}^{u}+X_{s}^{0}\right|}{(z-s)^{1+\alpha}} d s\right)^{2} \\
& \quad=: I_{1}^{u}(z)+I_{2}^{u}(z) .
\end{aligned}
$$

First, $I_{1}^{u}(z)$ admits the following bound:

$$
\begin{aligned}
I_{1}^{u}(z) \leq & C \mathrm{E} \sup _{t \in[0, z]}\left(\int_{0}^{t \wedge \tau^{u}}\left(a^{u}\left(s, X_{s}^{u}\right)-a^{0}\left(s, X_{s}^{0}\right)\right) d s\right)^{2} \\
& +C \mathrm{E} \sup _{t \in[0, z]}\left(\int_{0}^{t \wedge \tau^{u}}\left(b^{u}\left(s, X_{s}^{u}\right)-b^{0}\left(s, X_{s}^{0}\right)\right) d W_{s}\right)^{2} \\
& +C \mathrm{E} \sup _{t \in[0, z]}\left(\int_{0}^{t \wedge \tau^{u}}\left(c^{u}\left(s, X_{s}^{u}\right)-c^{0}\left(s, X_{s}^{0}\right)\right) d B_{s}^{H}\right)^{2} \\
= & : C\left(I_{1,1}^{u}(z)+I_{1,2}^{u}(z)+I_{1,3}^{u}(z)\right) .
\end{aligned}
$$

Second, by $(B 1)$ we have

$$
\begin{aligned}
I_{1,1}^{u}(z) \leq & C \mathrm{E}\left(\int_{0}^{z}\left|a^{u}\left(s, X_{s \wedge \tau^{u}}^{u}\right)-a^{u}\left(s, X_{s \wedge \tau^{u}}^{0}\right)\right| d s\right)^{2} \\
& +C \mathrm{E}\left(\int_{0}^{z}\left|a^{u}\left(s, X_{s}^{0}\right)-a^{0}\left(s, X_{s}^{0}\right)\right| d s\right)^{2} \\
\leq & C \int_{0}^{z} \mathrm{E}\left(X_{s \wedge \tau^{u}}^{u}-X_{s \wedge \tau^{u}}^{0}\right)^{2} d s+C\left(\alpha^{u}\right)^{2}\left(\mathrm{E} \int_{0}^{z}\left(1+\left|X_{s}^{0}\right|^{\nu_{1}}\right)^{2} d s\right) \\
\leq & C \int_{0}^{z} \mathrm{E} \sup _{q \in[0, s]}\left(X_{q \wedge \tau^{u}}^{u}-X_{q \wedge \tau^{u}}^{0}\right)^{2} d s+C\left(\alpha^{u}\right)^{2}\left(\mathrm{E} \int_{0}^{T}\left(1+\left|X_{s}^{0}\right|^{\nu_{1}}\right)^{2} d s\right) \\
\leq & C \int_{0}^{z} I_{1}^{u}(s) d s+C\left(\alpha^{u}\right)^{2} .
\end{aligned}
$$

The boundedness of the expectation

$$
\mathrm{E} \int_{0}^{T}\left(1+\left|X_{s}^{0}\right|^{\nu_{1}}\right)^{2} d s
$$


follows from Theorem 1 ;

$$
\begin{aligned}
\mathrm{E} \int_{0}^{T}\left(1+\left|X_{s}^{0}\right|^{\nu_{1}}\right)^{2} d s & \leq C \int_{0}^{T}\left(1+\left(\mathrm{E}\left(X_{s}^{0}\right)^{2}\right)^{\nu_{1}}\right) d s \\
& \leq C \int_{0}^{T}\left(1+\left(\left\|X^{0}\right\|_{\alpha}^{2}\right)^{\nu_{1}}\right) d s \leq C .
\end{aligned}
$$

Similarly we obtain the estimates

$$
\begin{aligned}
I_{1,2}^{u}(z) \leq & C \mathrm{E}\left(\int_{0}^{z}\left(b^{u}\left(s, X_{s \wedge \tau^{u}}^{u}\right)-b^{u}\left(s, X_{s \wedge \tau^{u}}^{0}\right)\right)^{2} d s\right) \\
& +C \mathrm{E}\left(\int_{0}^{z}\left(b^{u}\left(s, X_{s}^{0}\right)-b^{0}\left(s, X_{s}^{0}\right)\right)^{2} d s\right) \\
\leq & C \int_{0}^{z} \mathrm{E}\left(X_{s \wedge \tau^{u}}^{u}-X_{s \wedge \tau^{u}}^{0}\right)^{2} d s+C\left(\beta^{u}\right)^{2}\left(\mathrm{E} \int_{0}^{T}\left(1+\left|X_{s}^{0}\right|^{\nu_{1}}\right)^{2} d s\right) \\
\leq & C \int_{0}^{z} I_{1}^{u}(s) d s+C\left(\beta^{u}\right)^{2} .
\end{aligned}
$$

The term $I_{1,3}^{u}(z)$ is estimated by using the definition of the stopping time $\tau^{u}$ :

$$
\begin{aligned}
I_{1,3}^{u}(z)=\mathrm{E} \sup _{t \in[0, z]}\left(\int_{0}^{t \wedge \tau^{u}}\left(c^{u}\left(s, X_{s}^{u}\right)-c^{0}\left(s, X_{s}^{0}\right)\right) d B_{s}^{H}\right)^{2} \\
\leq C R^{2}\left(\mathrm{E} \sup _{t \in[0, z]}\left(\int_{0}^{t \wedge \tau^{u}} \frac{\left|c^{u}\left(s, X_{s}^{u}\right)-c^{0}\left(s, X_{s}^{0}\right)\right|}{s^{\alpha}} d s\right)^{2}\right. \\
\left.\quad+\mathrm{E} \sup _{t \in[0, z]}\left(\int_{0}^{t \wedge \tau^{u}} \int_{0}^{s} \frac{F_{1}(s, q)}{(s-q)^{1+\alpha}} d q d s\right)^{2}\right) \\
=: C R^{2}\left(I_{1,3,1}^{u}(z)+I_{1,3,2}^{u}(z)\right),
\end{aligned}
$$

where

$$
F_{1}(s, q)=\left|c^{u}\left(s, X_{s}^{u}\right)-c^{0}\left(s, X_{s}^{0}\right)-c^{u}\left(q, X_{q}^{u}\right)+c^{0}\left(q, X_{q}^{0}\right)\right| .
$$

Further we apply the Cauchy-Bunyakovskiǔ inequality:

$$
\begin{aligned}
I_{1,3,1}^{u}(z) & \leq C \mathrm{E} \sup _{t \in[0, z]}\left(\int_{0}^{t \wedge \tau^{u}}\left(c^{u}\left(s, X_{s}^{u}\right)-c^{0}\left(s, X_{s}^{0}\right)\right)^{2} d s\right) \\
& \leq C \mathrm{E} \int_{0}^{z}\left(X_{s \wedge \tau^{u}}^{u}-X_{s \wedge \tau^{u}}^{0}\right)^{2} d s+C\left(\gamma^{u}\right)^{2}\left(\mathrm{E} \int_{0}^{T}\left(1+\left|X_{s}^{0}\right|^{\nu_{1}}\right)^{2} d s\right) \\
& \leq C \int_{0}^{z} I_{1}^{u}(s) d s+C\left(\gamma^{u}\right)^{2},
\end{aligned}
$$

since $2 \alpha<1$.

Now we consider the term $I_{1,3,2}^{u}(z)$ :

$$
\begin{aligned}
I_{1,3,2}^{u}(z) \leq & C \mathrm{E}\left(\int_{0}^{z} \int_{0}^{s \wedge \tau^{u}} \frac{F_{2}(s, q)}{(s-q)^{1+\alpha}} d q d s\right)^{2} \\
& +C \mathrm{E} \sup _{t \in[0, z]}\left(\int_{0}^{t \wedge \tau^{u}} \int_{0}^{s} \frac{F_{3}(s, q)}{(s-q)^{1+\alpha}} d q d s\right)^{2} \\
:= & C\left(I_{1,3,2,1}^{u}(z)+I_{1,3,2,2}^{u}(z)\right)
\end{aligned}
$$


where

$$
\begin{gathered}
F_{2}(s, q)=\left|c^{u}\left(s, X_{s \wedge \tau^{u}}^{u}\right)-c^{u}\left(s, X_{s \wedge \tau^{u}}^{0}\right)-c^{u}\left(q, X_{q}^{u}\right)+c^{u}\left(q, X_{q}^{0}\right)\right|, \\
F_{3}(s, q)=\left|c^{u}\left(s, X_{s}^{0}\right)-c^{0}\left(s, X_{s}^{0}\right)-c^{u}\left(q, X_{q}^{0}\right)+c^{0}\left(q, X_{q}^{0}\right)\right| .
\end{gathered}
$$

We use Lemma 3 to estimate $I_{1,3,2,1}^{u}(z)$ :

$$
\begin{aligned}
I_{1,3,2,1}^{u}(z) \leq & C \mathrm{E}\left(\int_{0}^{z} \int_{0}^{s \wedge \tau^{u}} \frac{\left|X_{s \wedge \tau^{u}}^{u}-X_{s \wedge \tau^{u}}^{0}-X_{q}^{u}+X_{q}^{0}\right|}{(s-q)^{1+\alpha}} d q d s\right)^{2} \\
& +C \mathrm{E}\left(\int_{0}^{z} \int_{0}^{s \wedge \tau^{u}} \frac{\left|X_{s \wedge \tau^{u}}^{u}-X_{s \wedge \tau^{u}}^{0}\right|(s-q)^{\beta}}{(s-q)^{1+\alpha}} d q d s\right)^{2} \\
& +C \mathrm{E}\left(\int_{0}^{z} \int_{0}^{s \wedge \tau^{u}} \frac{F_{4}(s, q)}{(s-q)^{1+\alpha}} d q d s\right)^{2} \\
= & C\left(I_{1,3,2,1,1}^{u}(z)+I_{1,3,2,1,2}^{u}(z)+I_{1,3,2,1,3}^{u}(z)\right)
\end{aligned}
$$

where

$$
F_{4}(s, q)=\left|X_{s \wedge \tau^{u}}^{u}-X_{s \wedge \tau^{u}}^{0}\right|\left(\left|X_{s \wedge \tau^{u}}^{u}-X_{q}^{u}\right|^{\rho}+\left|X_{s \wedge \tau^{u}}^{0}-X_{q}^{0}\right|^{\rho}\right)
$$

and

$$
\begin{aligned}
I_{1,3,2,1,1}^{u}(z) & \leq C \int_{0}^{z} \mathrm{E}\left(\int_{0}^{s \wedge \tau^{u}} \frac{\left|X_{s \wedge \tau^{u}}^{u}-X_{s \wedge \tau^{u}}^{0}-X_{q}^{u}+X_{q}^{0}\right|}{(s-q)^{1+\alpha}} d q\right)^{2} d s \\
& \leq C \int_{0}^{z} I_{2}^{u}(s) d s, \\
I_{1,3,2,1,2}^{u}(z) & \leq C \int_{0}^{z} s^{2(\beta-\alpha)} \mathrm{E}\left(\left|X_{s \wedge \tau^{u}}^{u}-X_{s \wedge \tau^{u}}^{0}\right|\right)^{2} d s \leq C \int_{0}^{z} I_{1}^{u}(s) d s .
\end{aligned}
$$

Similarly to the proof of Theorem 3.2.5 in [3], one can show that

$$
\left|X_{t}^{u}-X_{s}^{u}\right| \leq C C_{T}^{u}(\omega, \delta) \exp \left\{C_{T}^{u}(\omega, \delta)^{1 /(1-\alpha)}\right\}|t-s|^{1 / 2-\delta_{1}}
$$

for all $u \in\left[0, u_{0}\right]$ and for all $\delta_{1} \in(0,1 / 2)$, where the process $C_{t}^{u}(\omega, \delta)$ is defined by relation (14). Recalling the assumption that $\tau^{u}=T \geq z$, we derive the inequality $C_{T}^{u}(\omega, \delta) \leq R$, whence

$$
\left|X_{t}^{u}-X_{s}^{u}\right| \leq C R \exp \left\{R^{1 /(1-\alpha)}\right\}|t-s|^{1 / 2-\delta_{1}} .
$$

Then

$$
\begin{aligned}
I_{1,3,2,1,3}^{u}(z) & \leq \mathrm{E}\left(\int_{0}^{z} \int_{0}^{s \wedge \tau^{u}} \frac{F_{5}(s, q)}{(s-q)^{1+\alpha}} d q d s\right)^{2} \\
& \leq C R^{2 \rho} \exp \left\{2 \rho R^{1 /(1-\alpha)}\right\} \int_{0}^{z} s^{\rho-2 \rho \delta_{1}-2 \alpha} \mathrm{E}\left(\left|X_{s \wedge \tau^{u}}^{u}-X_{s \wedge \tau^{u}}^{0}\right|\right)^{2} d s \\
& \leq C R^{2 \rho} \exp \left\{2 \rho R^{1 /(1-\alpha)}\right\} \int_{0}^{z} I_{1}^{u}(s) d s,
\end{aligned}
$$

where

$$
F_{5}(s, q)=\left|X_{s \wedge \tau^{u}}^{u}-X_{s \wedge \tau^{u}}^{0}\right|\left(2 R^{\rho} \exp \left\{\rho R^{1 /(1-\alpha)}\right\}|s-q|^{\rho\left(1 / 2-\delta_{1}\right)}\right)
$$

and $\delta_{1}$ is chosen such that $\rho-2 \rho \delta_{1}-2 \alpha>0$. This can be done, indeed, since $\alpha<\rho-1 / 2$ and thus $\rho-2 \alpha>1 / 2-\alpha>0$. 
Consider $I_{1,3,2,2}^{u}(z)$. We have

$$
\begin{aligned}
& I_{1,3,2,2}^{u}(z)=\mathrm{E} \sup _{t \in[0, z]}\left(\int_{0}^{t \wedge \tau^{u}} \int_{0}^{s} \frac{\left|d^{u}\left(s, X_{s}^{0}\right)-d^{u}\left(q, X_{q}^{0}\right)\right|}{(s-q)^{1+\alpha}} d q d s\right)^{2} \\
& \leq C \mathrm{E} \sup _{t \in[0, z]}\left(\int_{0}^{t \wedge \tau^{u}} \int_{0}^{s} \frac{\left|d^{u}\left(s, X_{s}^{0}\right)-d^{u}\left(s, X_{q}^{0}\right)\right|}{(s-q)^{1+\alpha}} d q d s\right)^{2} \\
& +C \mathrm{E} \sup _{t \in[0, z]}\left(\int_{0}^{t \wedge \tau^{u}} \int_{0}^{s} \frac{\left|d^{u}\left(s, X_{q}^{0}\right)-d^{u}\left(q, X_{q}^{0}\right)\right|}{(s-q)^{1+\alpha}} d q d s\right)^{2} \\
& \leq C \mathrm{E} \sup _{t \in[0, z]}\left(\int_{0}^{t \wedge \tau^{u}} \int_{0}^{s} \frac{\varkappa^{u}\left|X_{s}^{0}-X_{q}^{0}\right|^{\theta_{2}}}{(s-q)^{1+\alpha}} d q d s\right)^{2} \\
& +C \mathrm{E} \sup _{t \in[0, z]}\left(\int_{0}^{t \wedge \tau^{u}} \int_{0}^{s} \frac{\varphi^{u}(s-q)^{\theta_{1}}\left(1+\left|X_{q}^{0}\right|^{\nu_{2}}\right)}{(s-q)^{1+\alpha}} d q d s\right)^{2} \\
& \leq C\left(\varkappa^{u}\right)^{2} R^{2 \theta_{2}} \exp \left\{2 \theta_{2} R^{1 /(1-\alpha)}\right\} \mathrm{E}\left(\int_{0}^{T} \int_{0}^{s} \frac{(s-q)^{\theta_{2}\left(1 / 2-\delta_{1}\right)}}{(s-q)^{1+\alpha}} d q d s\right)^{2} \\
& +C\left(\varphi^{u}\right)^{2} \mathrm{E}\left(\int_{0}^{T} \int_{0}^{s} \frac{\left(1+\left|X_{q}^{0}\right|^{\nu_{2}}\right)}{(s-q)^{1+\alpha-\theta_{1}}} d q d s\right)^{2} \\
& \leq C\left(\varkappa^{u}\right)^{2} R^{2 \theta_{2}} \exp \left\{2 \theta_{2} R^{1 /(1-\alpha)}\right\} \\
& +C\left(\varphi^{u}\right)^{2} \mathrm{E}\left(\int_{0}^{T}\left(1+\left|X_{q}^{0}\right|^{\nu_{2}}\right)(t-q)^{\theta_{1}-\alpha} d q\right)^{2} \\
& \leq C\left(\varkappa^{u}\right)^{2} R^{2 \theta_{2}} \exp \left\{2 \theta_{2} R^{1 /(1-\alpha)}\right\}+C\left(\varphi^{u}\right)^{2},
\end{aligned}
$$

where $\delta_{1}$ satisfies an extra condition $\theta_{2}\left(1 / 2-\delta_{1}\right)-\alpha>0$. This can be achieved, indeed, since $\theta_{2}>2 \alpha$.

Thus

$$
\begin{aligned}
I_{1}^{u}(z) \leq & C\left(\alpha^{u}\right)^{2}+C\left(\beta^{u}\right)^{2}+C R^{2}\left(\gamma^{u}\right)^{2} \\
& +C R^{2+2 \theta_{2}} \exp \left\{2 \theta_{2} R^{1 /(1-\alpha)}\right\}\left(\varkappa^{u}\right)^{2}+C R^{2}\left(\varphi^{u}\right)^{2} \\
& +C R^{2+2 \rho} \exp \left\{2 \rho R^{1 /(1-\alpha)}\right\} \int_{0}^{z} I_{1}^{u}(s) d s+C R^{2} \int_{0}^{z} I_{2}^{u}(s) d s .
\end{aligned}
$$

Now we turn back to the term $I_{2}^{u}(z)$. This function admits the following bound:

$$
\begin{aligned}
I_{2}^{u}(z) \leq C\left(\mathrm{E}\left(\int_{0}^{z \wedge \tau^{u}} \frac{\int_{s}^{z \wedge \tau^{u}}\left(a^{u}\left(q, X_{q}^{u}\right)-a^{0}\left(q, X_{q}^{0}\right)\right) d q}{(z-s)^{1+\alpha}} d s\right)^{2}\right. \\
+\mathrm{E}\left(\int_{0}^{z \wedge \tau^{u}} \frac{\int_{s}^{z \wedge \tau^{u}}\left(b^{u}\left(q, X_{q}^{u}\right)-b^{0}\left(q, X_{q}^{0}\right)\right) d W_{q}}{(z-s)^{1+\alpha}} d s\right)^{2} \\
\left.+\mathrm{E}\left(\int_{0}^{z \wedge \tau^{u}} \frac{\int_{s}^{z \wedge \tau^{u}}\left(c^{u}\left(q, X_{q}^{u}\right)-c^{0}\left(q, X_{q}^{0}\right)\right) d B_{q}^{H}}{(z-s)^{1+\alpha}} d s\right)^{2}\right) \\
=: C\left(I_{2,1}^{u}(z)+I_{2,2}^{u}(z)+I_{2,3}^{u}(z)\right) .
\end{aligned}
$$


Next we estimate the term $I_{2,1}^{u}(z)$ :

$$
\begin{aligned}
I_{2,1}^{u}(z) \leq & C \mathrm{E}\left(\int_{0}^{z \wedge \tau^{u}} \frac{\int_{s}^{z \wedge \tau^{u}}\left(a^{u}\left(q, X_{q}^{u}\right)-a^{u}\left(q, X_{q}^{0}\right)\right) d q}{(z-s)^{1+\alpha}} d s\right)^{2} \\
& +C \mathrm{E}\left(\int_{0}^{z \wedge \tau^{u}} \frac{\int_{s}^{z \wedge \tau^{u}}\left(a^{u}\left(q, X_{q}^{0}\right)-a^{0}\left(q, X_{q}^{0}\right)\right) d q}{(z-s)^{1+\alpha}} d s\right)^{2} \\
= & C\left(I_{2,1,1}^{u}(z)+I_{2,1,2}^{u}(z)\right) .
\end{aligned}
$$

If $\gamma$ is such that $0<\alpha<\gamma<1 / 2$, then

$$
\begin{aligned}
I_{2,1,1}^{u}(z) & =\mathrm{E}\left(\int_{0}^{z \wedge \tau^{u}} \frac{\int_{s}^{z \wedge \tau^{u}}\left(a^{u}\left(q, X_{q}^{u}\right)-a^{u}\left(q, X_{q}^{0}\right)\right) d q}{(z-s)^{1+\alpha-\gamma}(z-s)^{\gamma}} d s\right)^{2} \\
& \leq C \mathrm{E} \int_{0}^{z \wedge \tau^{u}} \frac{(z-s) \int_{0}^{z \wedge \tau^{u}}\left|X_{q}^{u}-X_{q}^{0}\right|^{2} d q}{(z-s)^{2+2 \alpha-2 \gamma} d s} \\
& \leq C \int_{0}^{z}\left(X_{s \wedge \tau^{u}}^{u}-X_{s \wedge \tau^{u}}^{0}\right)^{2} d s \leq C \int_{0}^{z} I_{1}^{u}(s) d s
\end{aligned}
$$

Similarly

$$
\begin{aligned}
I_{2,1,2}^{u}(z) & \leq \mathrm{E}\left(\int_{0}^{z \wedge \tau^{u}} \frac{\int_{s}^{z \wedge \tau^{u}}\left(\alpha^{u}\left(1+\left|X_{q}^{0}\right|^{\nu_{1}}\right)\right) d q}{(z-s)^{1+\alpha}} d s\right)^{2} \\
& \leq C\left(\alpha^{u}\right)^{2}\left(\int_{0}^{z} \frac{(z-s) \int_{s}^{z} \mathrm{E}\left(1+\left|X_{q}^{0}\right|^{\nu_{1}}\right)^{2} d q}{(z-s)^{2+2 \alpha-2 \gamma}} d s\right) \leq C\left(\alpha^{u}\right)^{2} .
\end{aligned}
$$

Now we estimate $I_{2,2}^{u}(z)$ :

$$
\begin{aligned}
I_{2,2}^{u}(z) \leq & C \mathrm{E} \int_{0}^{z \wedge \tau^{u}} \frac{\int_{s}^{z \wedge \tau^{u}}\left(b^{u}\left(q, X_{q}^{u}\right)-b^{0}\left(q, X_{q}^{0}\right)\right)^{2} d q}{(z-s)^{2+2 \alpha-2 \gamma}} d s \\
\leq & C \mathrm{E} \int_{0}^{z \wedge \tau^{u}} \frac{\int_{s}^{z \wedge \tau^{u}}\left(b^{u}\left(q, X_{q}^{u}\right)-b^{u}\left(q, X_{q}^{0}\right)\right)^{2} d q}{(z-s)^{2+2 \alpha-2 \gamma} d s} \\
& +C \mathrm{E} \int_{0}^{z \wedge \tau^{u}} \frac{\int_{s}^{z \wedge \tau^{u}}\left(b^{u}\left(q, X_{q}^{0}\right)-b^{0}\left(q, X_{q}^{0}\right)\right)^{2} d q}{(z-s)^{2+2 \alpha-2 \gamma}} d s \\
= & C\left(I_{2,2,1}^{u}(z)+I_{2,2,2}^{u}(z)\right) .
\end{aligned}
$$

Then

$$
I_{2,2,1}^{u}(z) \leq C \mathrm{E} \int_{0}^{z} \frac{\int_{s}^{z}\left(X_{q \wedge \tau}^{u}-X_{q \wedge \tau}^{0}\right)^{2} d q}{(z-s)^{2+2 \alpha-2 \gamma}} d s \leq C \int_{0}^{z} \frac{I_{1}^{u}(q)}{(z-q)^{1+2 \alpha-2 \gamma}} d q
$$

and

$$
\begin{aligned}
I_{2,2,2}^{u}(z) & \leq \mathrm{E} \int_{0}^{z \wedge \tau^{u}} \frac{\int_{s}^{z \wedge \tau^{u}}\left(\left(\beta^{u}\right)^{2}\left(1+\left|X_{q}^{0}\right|^{\nu_{1}}\right)^{2}\right) d q}{(z-s)^{2+2 \alpha-2 \gamma}} d s \\
& \leq C\left(\beta^{u}\right)^{2} \int_{0}^{z} \frac{\int_{s}^{z} \mathrm{E}\left(1+\left|X_{q}^{0}\right|^{\nu_{1}}\right)^{2} d q}{(z-s)^{2+2 \alpha-2 \gamma}} d s \leq C\left(\beta^{u}\right)^{2} .
\end{aligned}
$$


Next we consider $I_{2,3}^{u}(z)$ :

$$
\begin{aligned}
I_{2,3}^{u}(z) \leq & C R^{2} \mathrm{E}\left(\int_{0}^{z \wedge \tau^{u}}\left(\int_{s}^{z \wedge \tau^{u}} \frac{c^{u}\left(q, X_{q}^{u}\right)-c^{0}\left(q, X_{q}^{0}\right)}{(q-s)^{\alpha}} d q\right)(z-s)^{-(1+\alpha)} d s\right)^{2} \\
& +C R^{2}\left(\mathrm{E} \int_{0}^{z \wedge \tau^{u}}\left(\int_{s}^{z \wedge \tau^{u}} \int_{s}^{q} \frac{F_{6}(v, q)}{(q-v)^{1+\alpha}} d v d q\right)^{2}(z-s)^{-(2+2 \alpha-2 \gamma)} d s\right) \\
= & : C R^{2}\left(I_{2,3,1}^{u}(z)+I_{2,3,2}^{u}(z)\right),
\end{aligned}
$$

where

$$
F_{6}(v, q)=\left|c^{u}\left(q, X_{q}^{u}\right)-c^{0}\left(q, X_{q}^{0}\right)-c^{u}\left(v, X_{v}^{u}\right)+c^{0}\left(v, X_{v}^{0}\right)\right| .
$$

The terms are estimated separately:

$$
\begin{aligned}
I_{2,3,1}^{u}(z) & \leq\left(\mathrm{E} \int_{0}^{z \wedge \tau^{u}}\left(\int_{s}^{z \wedge \tau^{u}} \frac{c^{u}\left(q, X_{q}^{u}\right)-c^{0}\left(q, X_{q}^{0}\right)}{(q-s)^{\alpha}} d q\right)(z-s)^{-(1+\alpha)} d s\right)^{2} \\
& \leq\left(\mathrm{E} \int_{0}^{z \wedge \tau^{u}}\left(c^{u}\left(q, X_{q}^{u}\right)-c^{0}\left(q, X_{q}^{0}\right)\right) \int_{0}^{q} \frac{1}{(q-s)^{\alpha}} \frac{1}{(z-s)^{1+\alpha}} d s d q\right)^{2} .
\end{aligned}
$$

Using inequality (4.15) of [1], we obtain

$$
\begin{aligned}
I_{2,3,1}^{u}(z) \leq & C\left(\mathrm{E} \int_{0}^{z \wedge \tau^{u}}\left(c^{u}\left(q, X_{q}^{u}\right)-c^{0}\left(q, X_{q}^{0}\right)\right) \frac{1}{(z-q)^{2 \alpha}} d q\right)^{2} \\
\leq & C \mathrm{E} \int_{0}^{z \wedge \tau^{u}} \frac{\left(c^{u}\left(s, X_{s}^{u}\right)-c^{0}\left(s, X_{s}^{0}\right)\right)^{2}}{(z-s)^{2 \alpha}} d s \\
\leq & C \mathrm{E} \int_{0}^{z \wedge \tau^{u}} \frac{\left(c^{u}\left(s, X_{s}^{u}\right)-c^{u}\left(s, X_{s}^{0}\right)\right)^{2}}{(z-s)^{2 \alpha}} d s \\
& +C \mathrm{E} \int_{0}^{z \wedge \tau^{u}} \frac{\left(c^{u}\left(s, X_{s}^{0}\right)-c^{0}\left(s, X_{s}^{0}\right)\right)^{2}}{(z-s)^{2 \alpha}} d s \\
\leq & C \int_{0}^{z} \frac{I_{1}^{u}(s)}{(z-s)^{2 \alpha}} d s+C\left(\gamma^{u}\right)^{2} .
\end{aligned}
$$

Next we estimate $I_{2,3,2}^{u}(z)$ :

$$
\begin{aligned}
I_{2,3,2}^{u}(z)= & \mathrm{E} \int_{0}^{z \wedge \tau^{u}}\left(\int_{s}^{z \wedge \tau^{u}} \int_{s}^{q} \frac{\left|c^{u}\left(q, X_{q}^{u}\right)-c^{0}\left(q, X_{q}^{0}\right)-c^{u}\left(v, X_{v}^{u}\right)+c^{0}\left(v, X_{v}^{0}\right)\right|}{(q-v)^{\alpha}} d v d q\right)^{2} \times(z-s)^{-(2+2 \alpha-2 \gamma)} d s \\
\leq & C \mathrm{E} \int_{0}^{z \wedge \tau^{u}}\left(\int_{s}^{z \wedge \tau^{u}} \int_{s}^{q} \frac{F_{7}(v, q)}{(q-v)^{\alpha}} d v d q\right)^{2}(z-s)^{-(2+2 \alpha-2 \gamma)} d s \\
& +C \mathrm{E} \int_{0}^{z \wedge \tau^{u}}\left(\int_{s}^{z \wedge \tau^{u}} \int_{s}^{q} \frac{F_{8}(v, q)}{(q-v)^{\alpha}} d v d q\right)^{2}(z-s)^{-(2+2 \alpha-2 \gamma)} d s \\
= & : C\left(I_{2,3,2,1}^{u}(z)+I_{2,3,2,2}^{u}(z)\right),
\end{aligned}
$$

where

$$
\begin{aligned}
& F_{7}(v, q)=\left|c^{u}\left(q, X_{q}^{u}\right)-c^{u}\left(q, X_{q}^{0}\right)-c^{u}\left(v, X_{v}^{u}\right)+c^{u}\left(v, X_{v}^{0}\right)\right|, \\
& F_{8}(v, q)=\left|c^{u}\left(q, X_{q}^{0}\right)-c^{0}\left(q, X_{q}^{0}\right)-c^{u}\left(v, X_{v}^{0}\right)+c^{0}\left(v, X_{v}^{0}\right)\right| .
\end{aligned}
$$


Using Lemma 3 we estimate $I_{2,3,2,1}^{u}(z)$ :

$$
\begin{aligned}
I_{2,3,2,1}^{u}(z) \leq & C \mathrm{E} \int_{0}^{z \wedge \tau^{u}}\left(\int_{s}^{z \wedge \tau^{u}} \int_{s}^{q} \frac{\left|X_{q}^{u}-X_{q}^{0}-X_{v}^{u}+X_{v}^{0}\right|}{(q-v)^{1+\alpha}} d v d q\right)^{2}(z-s)^{-(2+2 \alpha-2 \gamma)} d s \\
& +C \mathrm{E} \int_{0}^{z \wedge \tau^{u}}\left(\int_{s}^{z \wedge \tau^{u}} \int_{s}^{q} \frac{\left|X_{q}^{u}-X_{q}^{0}\right|(q-v)^{\beta}}{(q-v)^{1+\alpha}} d v d q\right)^{2}(z-s)^{-(2+2 \alpha-2 \gamma)} d s \\
& +C \mathrm{E} \int_{0}^{z \wedge \tau^{u}}\left(\int_{s}^{z \wedge \tau^{u}} \int_{s}^{q} \frac{\left|X_{q}^{u}-X_{q}^{0}\right|\left(\left|X_{q}^{u}-X_{v}^{u}\right|^{\rho}+\left|X_{q}^{0}-X_{v}^{0}\right|^{\rho}\right)}{(q-v)^{1+\alpha}} d v d q\right)^{2} \\
= & : C\left(I_{2,3,2,1,1}^{u}(z)+I_{2,3,2,1,2}^{u}(z)+I_{2,3,2,1,3}^{u}(z)\right) .
\end{aligned}
$$

Then we estimate $I_{2,3,2,1,1}^{u}(z)$ by taking into account that $2 \gamma-2 \alpha>0$ :

$I_{2,3,2,1,1}^{u}(z)$

$$
\begin{aligned}
& \leq C \mathrm{E} \int_{0}^{z} \int_{s}^{z}\left(\int_{s}^{q \wedge \tau^{u}} \frac{\left|X_{q \wedge \tau^{u}}^{u}-X_{q \wedge \tau^{u}}^{0}-X_{v}^{u}+X_{v}^{0}\right|}{(q-v)^{1+\alpha}} d v\right)^{2} d q(z-s)^{-(1+2 \alpha-2 \gamma)} d s \\
& \leq C \mathrm{E} \int_{0}^{z}\left(\int_{0}^{q \wedge \tau^{u}} \frac{\left|X_{q \wedge \tau^{u}}^{u}-X_{q \wedge \tau^{u}}^{0}-X_{v}^{u}+X_{v}^{0}\right|}{(q-v)^{1+\alpha}} d v\right)^{2} d q \\
& \leq C \int_{0}^{z} I_{2}^{u}(s) d s
\end{aligned}
$$

and

$$
\begin{aligned}
I_{2,3,2,1,2}^{u}(z) & \leq \mathrm{E} \int_{0}^{z \wedge \tau^{u}} \frac{\left(\int_{s}^{z \wedge \tau^{u}}\left|X_{q}^{u}-X_{q}^{0}\right|(q-s)^{\beta-\alpha} d q\right)^{2}}{(z-s)^{2+2 \alpha-2 \gamma}} d s \\
& \leq C \mathrm{E} \int_{0}^{z \wedge \tau^{u}} \frac{(z-s)^{1+2 \beta-2 \alpha} \int_{s}^{z \wedge \tau^{u}}\left(X_{q}^{u}-X_{q}^{0}\right)^{2} d q}{(z-s)^{2+2 \alpha-2 \gamma}} d s \\
& \leq C \mathrm{E} \int_{0}^{z} \frac{\int_{s}^{z}\left(X_{q \wedge \tau^{u}}^{u}-X_{q \wedge \tau^{u}}^{0}\right)^{2} d q}{(z-s)^{1+4 \alpha-2 \beta-2 \gamma}} d s \leq C \int_{0}^{z} I_{1}^{u}(s) d s .
\end{aligned}
$$

The latter inequality is obtained by changing the order of integration and in view of the inequality $2 \beta+2 \gamma-4 \alpha>0$. Finally,

$I_{2,3,2,1,3}^{u}(z)$

$$
\begin{aligned}
& \leq C R^{2 \rho} \exp \left\{2 \rho R^{1 /(1-\alpha)}\right\} \mathrm{E} \int_{0}^{z \wedge \tau^{u}}\left(\int_{s}^{z \wedge \tau^{u}} \int_{s}^{q} \frac{\left|X_{q}^{u}-X_{q}^{0}\right|(q-v)^{\rho\left(1 / 2-\delta_{1}\right)}}{(q-v)^{1+\alpha}} d v d q\right)^{2} \\
& \times(z-s)^{-(2+2 \alpha-2 \gamma)} d s \\
& \leq C R^{2 \rho} \exp \left\{2 \rho R^{1 /(1-\alpha)}\right\} \mathrm{E} \int_{0}^{z \wedge \tau^{u}} \frac{\left(\int_{s}^{z \wedge \tau^{u}}\left|X_{q}^{u}-X_{q}^{0}\right|(q-s)^{\rho\left(1 / 2-\delta_{1}\right)-\alpha} d q\right)^{2}}{(z-s)^{2+2 \alpha-2 \gamma}} d s \\
& \leq C R^{2 \rho} \exp \left\{2 \rho R^{1 /(1-\alpha)}\right\} \mathrm{E} \int_{0}^{z \wedge \tau^{u}} \frac{(z-s) \int_{0}^{z}\left(X_{q \wedge \tau}^{u}-X_{q \wedge \tau}^{0}\right)^{2} d q}{(z-s)^{2+2 \alpha-2 \gamma}} d s \\
& \leq C R^{2 \rho} \exp \left\{2 \rho R^{1 /(1-\alpha)}\right\} \int_{0}^{z} I_{1}^{u}(s) d s .
\end{aligned}
$$


As above, $0<\delta_{1}<1 / 2-\alpha / \rho$ and $\alpha<\gamma<1 / 2$ in the latter relation.

Now we turn back to $I_{2,3,2,2}^{u}(z)$ :

$$
\begin{aligned}
I_{2,3,2,2}^{u}(z)=\mathrm{E} \int_{0}^{z \wedge \tau^{u}}\left(\int_{s}^{z \wedge \tau^{u}} \int_{s}^{q} \frac{\left|d^{u}\left(q, X_{q}^{0}\right)-d^{u}\left(v, X_{v}^{0}\right)\right|}{(q-v)^{\alpha}} d v d q\right)^{2}(z-s)^{-(2+2 \alpha-2 \gamma)} d s \\
\leq C \mathrm{E} \int_{0}^{z \wedge \tau^{u}}\left(\int_{s}^{z \wedge \tau^{u}} \int_{s}^{q} \frac{\left|d^{u}\left(q, X_{q}^{0}\right)-d^{u}\left(q, X_{v}^{0}\right)\right|}{(q-v)^{\alpha}} d v d q\right)^{2}(z-s)^{-(2+2 \alpha-2 \gamma)} d s \\
+C \mathrm{E} \int_{0}^{z \wedge \tau^{u}}\left(\int_{s}^{z \wedge \tau^{u}} \int_{s}^{q} \frac{\left|d^{u}\left(q, X_{v}^{0}\right)-d^{u}\left(v, X_{v}^{0}\right)\right|}{(q-v)^{\alpha}} d v d q\right)^{2}(z-s)^{-(2+2 \alpha-2 \gamma)} d s \\
=: C\left(I_{2,3,2,2,1}^{u}(z)+I_{2,3,2,2,2}^{u}(z)\right) .
\end{aligned}
$$

The latter two terms are estimated as follows:

$$
\begin{aligned}
& I_{2,3,2,2,1}^{u}(z) \leq \mathrm{E} \int_{0}^{z \wedge \tau^{u}}\left(\int_{s}^{z \wedge \tau^{u}} \int_{s}^{q} \frac{\varkappa^{u}\left|X_{q}^{0}-X_{v}^{0}\right|^{\theta_{2}}}{(q-v)^{1+\alpha}} d v d q\right)^{2}(z-s)^{-(2+2 \alpha-2 \gamma)} d s \\
& \leq C R^{2 \theta_{2}} \exp \left\{2 \theta_{2} R^{1 /(1-\alpha)}\right\}\left(\varkappa^{u}\right)^{2} \\
& \times \mathrm{E} \int_{0}^{z \wedge \tau^{u}}\left(\int_{s}^{z \wedge \tau^{u}} \int_{s}^{q} \frac{(q-v)^{\theta_{2}\left(1 / 2-\delta_{1}\right)}}{(q-v)^{1+\alpha}} d v d q\right)^{2} \frac{1}{(z-s)^{2+2 \alpha-2 \gamma}} d s \\
& \leq C R^{2 \theta_{2}} \exp \left\{2 \theta_{2} R^{1 /(1-\alpha)}\right\}\left(\varkappa^{u}\right)^{2} \mathrm{E} \int_{0}^{z \wedge \tau^{u}} \frac{\left(\int_{s}^{z \wedge \tau^{u}}(q-s)^{\theta_{2}\left(1 / 2-\delta_{1}\right)-\alpha} d q\right)^{2}}{(z-s)^{2+2 \alpha-2 \gamma}} d s \\
& \leq C R^{\theta_{2}} \exp \left\{2 \theta_{2} R^{1 /(1-\alpha)}\right\}\left(\varkappa^{u}\right)^{2} \int_{0}^{z} \frac{1}{(z-s)^{2 \alpha-2 \gamma}} d s \\
& \leq C R^{2 \theta_{2}} \exp \left\{2 \theta_{2} R^{1 /(1-\alpha)}\right\}\left(\varkappa^{u}\right)^{2}
\end{aligned}
$$

for $\theta_{2}\left(\frac{1}{2}-\delta_{1}\right)-\alpha>0$. Such a choice is possible, since $\theta_{2}>2 \alpha$. Choosing

$$
\delta_{1} \in\left(0, \min \left\{\frac{1}{2}-\frac{\alpha}{\rho}, \frac{1}{2}-\frac{\alpha}{\theta_{2}}\right\}\right),
$$

we conclude that

$$
\begin{aligned}
I_{2,3,2,2,2}^{u}(z) & \leq \mathrm{E} \int_{0}^{z \wedge \tau^{u}} \frac{\left(\int_{s}^{z \wedge \tau^{u}} \int_{s}^{q} \frac{\varphi^{u}(q-v)^{\theta_{1}}\left(1+\left|X_{v}^{0}\right|^{\nu_{2}}\right)}{(q-v)^{1+\alpha}} d v d q\right)^{2}}{(z-s)^{2+2 \alpha-2 \gamma}} d s \\
& \leq C\left(\varphi^{u}\right)^{2} \int_{0}^{z} \frac{(z-s) \int_{s}^{z} \mathrm{E}\left(1+\left|X_{v}^{0}\right|^{\nu_{2}}\right)^{2} d v}{(z-s)^{2+2 \alpha-2 \gamma}} \\
& \leq C\left(\varphi^{u}\right)^{2} .
\end{aligned}
$$

Since $\alpha<\gamma<1 / 2$, we have

$$
\begin{aligned}
I_{2}^{u}(z) \leq & C\left(\alpha^{u}\right)^{2}+C\left(\beta^{u}\right)^{2}+C R^{2}\left(\gamma^{u}\right)^{2}+C R^{2+2 \theta_{2}} \exp \left\{2 \theta_{2} R^{1 /(1-\alpha)}\right\}\left(\varkappa^{u}\right)^{2} \\
& +C R^{2}\left(\varphi^{u}\right)^{2}+C R^{2+2 \rho} \exp \left\{2 \rho R^{1 /(1-\alpha)}\right\} \int_{0}^{z} \frac{I_{1}^{u}(s)}{(z-s)^{1+2 \alpha-2 \gamma}} d s \\
& +C R^{2} \int_{0}^{z} I_{2}^{u}(s) d s .
\end{aligned}
$$


We obtain from (19) and (20) that

$$
\begin{aligned}
& I_{1}^{u}(z)+I_{2}^{u}(z) \\
& \leq C\left(\left(\alpha^{u}\right)^{2}+\left(\beta^{u}\right)^{2}+R^{2}\left(\gamma^{u}\right)^{2}\right. \\
& \quad+R^{2+2 \theta_{2}} \exp \left\{2 \theta_{2} R^{1 /(1-\alpha)}\right\}\left(\varkappa^{u}\right)^{2}+R^{2}\left(\varphi^{u}\right)^{2} \\
& \left.\quad+R^{2+2 \rho} \exp \left\{2 \rho R^{1 /(1-\alpha)}\right\}\left(\int_{0}^{z} \frac{I_{1}^{u}(s)}{(z-s)^{1+2 \alpha-2 \gamma}} d s+\int_{0}^{z} I_{2}^{u}(s) d s\right)\right) .
\end{aligned}
$$

The modified Gronwall lemma (see [1]) implies that

$$
\begin{aligned}
I_{1}^{u}(z)+ & I_{2}^{u}(z) \\
\leq & C R^{2+2 \theta_{2}} \exp \left\{2 \theta_{2} R^{1 /(1-\alpha)}\right\}\left(\left(\alpha^{u}\right)^{2}+\left(\beta^{u}\right)^{2}+\left(\gamma^{u}\right)^{2}+\left(\varkappa^{u}\right)^{2}+\left(\varphi^{u}\right)^{2}\right) \\
& \times \exp \left\{z\left(C R^{2+2 \rho} \exp \left\{2 \rho R^{1 /(1-\alpha)}\right\}\right)^{1 /(2 \gamma-2 \alpha)}\right\}
\end{aligned}
$$

for all $z \in[0, T]$, whence

$$
\begin{aligned}
I_{1}^{u}(T)= & \mathrm{E}\left(\sup _{t \in[0, T]}\left|X_{t \wedge \tau}^{u}-X_{t \wedge \tau}^{0}\right|\right)^{2} \\
\leq & C R^{2+2 \theta_{2}} \exp \left\{2 \theta_{2} R^{1 /(1-\alpha)}\right\} \\
& \times\left(\left(\alpha^{u}\right)^{2}+\left(\beta^{u}\right)^{2}+\left(\gamma^{u}\right)^{2}+\left(\varkappa^{u}\right)^{2}+\left(\varphi^{u}\right)^{2}\right) \\
& \times \exp \left\{T\left(C R^{2+2 \rho} \exp \left\{2 \rho R^{1 /(1-\alpha)}\right\}\right)^{1 /(2 \gamma-2 \alpha)}\right\} .
\end{aligned}
$$

Relations (17) and (23) yield

$$
\begin{aligned}
& \mathrm{P}\left(\sup _{t \in[0, T]}\left|X_{t \wedge \tau^{u}}^{u}-X_{t \wedge \tau^{u}}^{0}\right|>\varepsilon\right) \\
& \leq \frac{1}{\varepsilon^{2}} C R^{2+2 \theta_{2}} \exp \left\{2 \theta_{2} R^{1 /(1-\alpha)}\right\} \\
& \times\left(C\left(\alpha^{u}\right)^{2}+C\left(\beta^{u}\right)^{2}+C\left(\gamma^{u}\right)^{2}+C\left(\varkappa^{u}\right)^{2}+C\left(\varphi^{u}\right)^{2}\right) \\
& \times \exp \left\{T\left(C R^{2+2 \rho} \exp \left\{2 \rho R^{1 /(1-\alpha)}\right\}\right)^{1 /(2 \gamma-2 \alpha)}\right\} .
\end{aligned}
$$

Passing to the limit as $u \rightarrow 0$, we prove that the right hand side of (24) approaches 0 . Then (16) gives

$$
\limsup _{u \rightarrow 0+} \mathrm{P}\left(\sup _{t \in[0, T]}\left|X_{t}^{u}-X_{t}^{0}\right|>\varepsilon\right) \leq \limsup _{u \rightarrow 0+} \mathrm{P}\left(\tau^{u}<T\right) .
$$

Letting $R \rightarrow+\infty$, we obtain

$$
\limsup _{u \rightarrow 0+} \mathrm{P}\left(\sup _{t \in[0, T]}\left|X_{t}^{u}-X_{t}^{0}\right|>\varepsilon\right)=0 .
$$




\section{CONCLUding REMARKS}

A one-dimensional stochastic differential equation driven by both a standard Brownian motion and a fractional Brownian motion is considered in the paper. The coefficients of the equation are assumed to be nonhomogeneous. The coefficients as well as a random initial condition depend on a certain parameter $u \in\left[0, u_{0}\right]$. Conditions on the coefficients and on the initial conditions as functions of the parameter are found under which the sequence of solutions of stochastic differential equations as functions of the parameter $u$ converge in probability as $u \rightarrow 0$ to a solution of the limit equation.

\section{BIBLIOGRAPHY}

1. D. Nualart and A. Răşcanu, Differential equation driven by fractional Brownian motion, Collect. Math. 53 (2002), no. 1, 55-81. MR1893308(2003f:60105)

2. Yu. S. Mishura, Stochastic Calculus for Fractional Brownian Motion and Related Processes, Lecture Notes in Mathematics, Springer-Verlag, Berlin, 2008. MR2378138 (2008m:60064)

3. Yu. S. Mishura and S. V. Posashkov, Existence and uniqueness of solution of mixed stochastic differential equation driven by fractional Brownian motion and Wiener process, Theory Stoch. Process. 29 (2007), 152-165. MR2343820 (2009c:60158)

4. S. G. Samko, A. A. Kilbas, and O. I. Marichev, Fractional Integrals and Derivatives. Theory and Applications, Gordon and Breach Science Publishers, Yverdon, 1993. MR.1347689(96d:26012)

5. M. Zähle, Integration with respect to fractal functions and stochastic calculus. I, Probab. Theory Related Fields 111 (1988), no. 3, 333-374. MR1640795 (99j:60073)

Department of Probability Theory, Statistics, and Actuarial Mathematics, Faculty for Mechanics and Mathematics, National Taras Shevchenko University, Academician Glushkov Avenue 2, Kiev 03127, Ukraine

E-mail address: myus@univ.kiev.ua

Department of Probability Theory, Statistics, and Actuarial Mathematics, Faculty for Mechanics and Mathematics, National Taras Shevchenko University, ACademician Glushkov Avenue 2, Kiev 03127, Ukraine

E-mail address: revan1988@gmail.com

Department of Probability Theory, Statistics, and Actuarial Mathematics, Faculty for Mechanics and Mathematics, National Taras Shevchenko University, Academician Glushkov Avenue 2, Kiev 03127, Ukraine

E-mail address: corlagon@univ.kiev.ua 